\title{
Spherical brushes within spherical cavities: A self-consistent field and Monte Carlo study
}

\author{
Juan J. Cerdà, ${ }^{1, a)}$ Tomás Sintes, $^{2}$ and Raúl Toral ${ }^{2}$ \\ ${ }_{1}^{1}$ Institute for Computational Physics, Universität Stuttgart, 70569 Stuttgart, Germany \\ ${ }^{2}$ Instituto de Física Interdisciplinar y Sistemas Complejos IFISC (CSIC-UIB), \\ Universitat de les Illes Balears, 07122 Palma de Mallorca, Spain
}

(Received 1 July 2009; accepted 7 September 2009; published online 1 October 2009)

\begin{abstract}
We present an extensive numerical study on the behavior of spherical brushes confined into a spherical cavity. Self-consistent field (SCF) and off-lattice Monte Carlo (MC) techniques are used in order to determine the monomer and end-chain density profiles and the cavity pressure as a function of the brush properties. A comparison of the results obtained via SCF, MC, and the Flory theory for polymer solutions reveals SCF calculations to be a valuable alternative to MC simulations in the case of free and softly compressed brushes, while the Flory's theory accounts remarkably well for the pressure in the strongly compressed regime. In the range of high compressions, we have found the cavity pressure $P$ to follow a scale relationship with the monomer volume fraction $v$, $P \sim v^{\alpha}$. SCF calculations give $\alpha=2.15 \pm 0.05$, whereas MC simulations lead to $\alpha=2.73 \pm 0.04$. The underestimation of $\alpha$ by the SCF method is explained in terms of the inappropriate account of the monomer density correlations when a mean field approach is used. () 2009 American Institute of Physics. [doi:10.1063/1.3238568]
\end{abstract}

\section{INTRODUCTION}

Large sets of polymer chains terminally anchored or end grafted to a surface (known as "polymer brushes") are relevant in many areas of polymer science and technology ranging from medical and biological applications ${ }^{1-3}$ to nanotechnology ${ }^{4-7}$ and responsive systems. ${ }^{8-10}$ Polymer brushes anchored to planar surfaces were the first to be studied and characterized. Since the seminal works of de Gennes $^{11}$ and Alexander ${ }^{12}$ a remarkable progress has been done in the understanding and design of new applications involving flat brushes. ${ }^{13-24}$

In a subsequent stage polymer brushes grafted at curved interfaces either concave ${ }^{25-31}$ or convex ${ }^{32-35}$ have been studied. In concave (respectively convex) brushes the volume available to the polymer chains decreases (respectively increases), as the chains move away from the interface. A convex case of particular interest are spherical particles with polymer(polyelectrolyte) chains attached to their outer surfaces. Such systems are known as spherical polymer(polyelectrolyte) brushes, hairy particles or star polymer brushes. Spherical polymer brushes are very interesting systems due to their multiple possible uses: drug delivering, ${ }^{1,36}$ stabilization of colloidal particles, ${ }^{37,38}$ mimicking of hard spheres, ${ }^{39}$ creation of bioinspired materials, ${ }^{40}$ smart catalyst, ${ }^{9}$ etc. The study and characterization of polymer and polyelectrolyte spherical brushes have received considerable attention including experimental works, ${ }^{41,42}$ theoretical ${ }^{33,43,44}$ and selfconsistent field (SCF) developments, ${ }^{44-51}$ as well as numerical simulations. ${ }^{17,32,42,52-58}$ The determination of the pairwise interaction $^{59-64}$ and the study of solutions of spherical

${ }^{\text {a)} E l e c t r o n i c ~ m a i l: ~ j c e r d a @ i c p . u n i-s t u t t g a r t . d e . ~}$ brushes ${ }^{65-68}$ have also been studied extensively. Nonetheless, despite the large insight obtained from these works, the behavior of spherical brushes in constrained geometries is still poorly understood and has only been addressed in few works: the behavior of spherical brushes and star polymers within slit geometries has been recently addressed by Romiszowski-Sikorski, ${ }^{69}$ while the behavior of concave spherical brushes and three layer onion type micelles has been studied by Prochazka and co-workers. ${ }^{26,27,70}$ To our best knowledge, the behavior and properties of simple spherical convex brushes confined within spherical bare cavities have not been investigated yet. In addition to the academic interest, the knowledge about how convex brushes behave within a cavity can also provide a helpful insight into the understanding of nanobrush particles encapsulated within composite materials, ${ }^{6}$ the preparation of hybrid materials within mini- and micro-emulsions, ${ }^{71-73}$ as well as in the study of sterically stabilized liposomes and other hairy particles when trapped by macrophages within the body during endocytosis processes. ${ }^{1}$

The aim of this work is to study numerically the conformations and the forces that arise when a neutral spherical brush is confined within a spherical cavity as a function of the properties of the brush (length of the chains, grafting density, etc.) and the size of the cavity, as well as to develop tools that can help easily predict the value of such forces and pressure inside the cavity. In order to understand the conformations and forces that arise in a spherical brush under isotropic compression, the forces and pressures obtained from Monte Carlo (MC) simulations are compared to two alternative approaches: SCF calculations and the Flory theory for polymer solutions. The comparison will allow us to test and discuss the performance of such alternative approaches. 


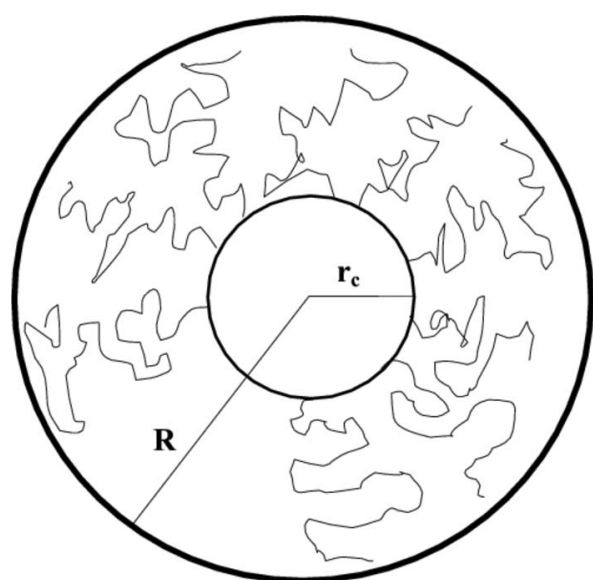

FIG. 1. Schematic representation of a spherical brush with an impenetrable core of radius $r_{c}$ inside a spherical cavity of radius $R$.

The rest of the paper is organized as follows. In Sec. II we describe the MC simulations and the self-consistent method including a brief discussion about the calculation of the forces acting on the wall of the cavity. In Sec. III, we present a detailed analysis of the density profiles, forces, and the pressure inside the cavity using the three different approaches (MC, SCF, and Flory theory). Section IV concludes with a brief discussion and outline of the main results.

\section{NUMERICAL AND THEORETICAL APPROACHES}

\section{A. Monte Carlo method}

In order to simulate the interaction between a spherical brush confined inside a spherical cavity wall, we have used a three-dimensional off-lattice MC method. We have generated the brush by homogeneously distributing $f$ polymer chains grafted onto an impenetrable spherical surface of radius $r_{c}$. The cavity wall is also impenetrable with a variable radius $R$. The polymer chain is represented by the pearl necklace model ${ }^{74}$ containing $N$ beads of diameter $\sigma$. The distance between the centers of two consecutive beads in the chain is set to $1.1 \sigma$. The initial configuration of the self-avoiding polymer is randomly generated being the first monomer permanently anchored to the surface (it is never allowed to move). A schematic representation of the system is shown in Fig. 1.

Monomers interact through a steric hard-core potential of the form

$$
U_{\text {steric }}=\sum_{i, j=1}^{N \times f} u\left(r_{i j}\right)
$$

where $u$ is a hard sphere potential

$$
u\left(r_{i j}\right)= \begin{cases}0 & \text { for }\left|\mathbf{r}_{i}-\mathbf{r}_{j}\right|>\sigma \\ \infty & \text { for }\left|\mathbf{r}_{i}-\mathbf{r}_{j}\right|<\sigma\end{cases}
$$

In what follows we use dimensionless units such that $k T=1$.

Different polymer configurations are generated by changing the position of a randomly selected monomer. If the monomer is located inside the chain (between the first monomer, permanently anchored, and the last one) its position changes by rotating an arbitrary angle between 0 and $2 \pi$ around the axis connecting the previous and following monomers in the chain. Chain ends just perform random wiggling motions. The proposed motion is accepted if the excluded volume interaction is preserved [Eq. (2)]. A linkcell method ${ }^{75}$ has been implemented in the algorithm to efficiently check all possible monomer overlaps. We define one Monte Carlo step (MCS) as $N \times f$ trials to perform monomer moves.

Initially, the radius of the cavity wall is set to be larger than the maximum extent of the brush to ensure that during the equilibration process no interaction between the brush and the cavity wall occurs. The free spherical brush is equilibrated typically during $5 \times 10^{5} \mathrm{MCS}$. After this initial equilibration time, magnitudes of interest are recorded every 10 MCS. We now proceed to compress the brush. We reduce slowly the radius of the cavity using steps of $\delta R \sim 10^{-3}$. After each step we check if there are some monomers laying out of the surface. If that is the case, we revert to the size of the previous step and the system is run for 10 MCS before trying to reduce the radius to another $\delta R$. The size of the external cavity is smoothly reduced until we reach a radius size where measurements should be performed (typically each time $R$ decreases by one unit). At this point a further equilibration of $6 \times 10^{5} \mathrm{MCS}$ is performed before measurements for the cavity of size $R$ take place.

The force $F$ that the system exerts to avoid compression is computed as the change in the free energy $\mathcal{F}$ due to an infinitesimal change in the cavity radius $R$,

$$
F(R) \equiv-\frac{\partial \mathcal{F}(R)}{\partial R} .
$$

The free energy can be computed as $\mathcal{F}(R)=-\ln Z(R)$ with a partition function

$$
Z(R)=\int \prod_{i=1}^{N \times f} d \mathbf{r}_{i} \exp \left(-\sum_{j=1}^{N \times f} u\left(\mathbf{r}_{i j}\right)\right) .
$$

Due to the hard-core structure of the potential, see Eq. (2), the partition function $Z(R)$ is equal to the volume of the region of the configuration space $\Omega(R)$ of all possible polymer chain configurations compatible with the steric requirements. We now relate $Z(R)$ to the probability of compression of the system. ${ }^{16,76}$ Let $P_{C}(R)$ be the probability that the outer surface radius can be reduced from $R$ to $R-\delta R$. Due to the steric interactions, compression is possible if no monomer lies between distances $R-\delta R$ and $R$ from the center of the cavity. The final configuration, after compression, corresponds to an allowed configuration of $\Omega(R-\delta R)$. The compression probability is hence the ratio of volumes $P_{C}(R)$ $=Z(R-\delta R) / Z(R)$ and, in the limit of small $\delta R$, the force can be computed as

$$
F(R)=\frac{\partial \ln Z(R)}{\partial R} \approx \frac{1}{\delta R} \ln \frac{Z(R)}{Z(R-\delta R)}=\frac{-1}{\delta R} \ln P_{C}(R) .
$$

$P_{C}(R)$ is computed as the average value (typically we average over samples taken each $10 \mathrm{MCS}$ over $6 \times 10^{5} \mathrm{MCS}$ ) of the fraction of configurations for which compression would be possible. After a good estimator of $P_{C}(R)$ has been obtained, the system is slowly compressed until the cavity reaches a size where new measurements should be per- 
formed. The process is repeated until a desired minimum value of the radius $R$ is reached.

The monomer radial density $\phi$ and the end-chain radial density $\epsilon$ are defined as usual,

$$
\phi(r)=\frac{n(r)}{4 \pi r^{2} d r}, \quad \epsilon(r)=\frac{n_{E}(r)}{4 \pi r^{2} d r},
$$

$n(r)$ [respectively $\left.n_{E}(r)\right]$ being the number of monomers (respectively end-chain monomers) located between distances $r$ and $r+d r$ from the center of the sphere. The definitions are such that the following normalization conditions hold:

$$
\int_{0}^{\infty} d \mathbf{r} \phi(r)=N f, \quad \int_{0}^{\infty} d \mathbf{r} \epsilon(r)=N .
$$

\section{B. Self-consistent field method}

In order to compute the probability density function (pdf) for polymer systems, it is customary to derive a Schrödinger-like equation for the $\operatorname{pdf} G_{n}\left(\mathbf{h}^{\prime}, \mathbf{h}\right)$ for a single chain. ${ }^{77,78}$ The use of this formalism is a valid approximation for spatial scales much larger than the polymer blob size. However, for encapsulated polymer brushes, and mainly at moderate and high compressions, the spatial scale of the cavity is comparable to the blob size. Thus, the use of the precedent formalism to compute the pdf becomes inadequate. Instead, we compute the pdf of a polymer chain using directly the recurrence law for the pdf from which the Schrödinger-like equation derives. ${ }^{78}$ We must be aware that the use of the recurrence law still implies some approximations. It is assumed that on each monomer of the polymer chain acts a potential only depends on the position of the monomer in the system, $U=U(\mathbf{r})$, therefore, bond correlations are not taken into account. It is also assumed that the potential is a function of the local monomer density $U(\phi(\mathbf{r}))$, ignoring particle density correlations. Finally, the properties of the whole ensemble of chains are deduced from the pdf of a single chain.

Under the above assumptions, the spherical cavity is split in concentric shells of thickness $d r$ and all monomers inside a shell are assumed to be equivalent. A polymer chain composed of $N$ monomers is represented as a path of $N$ segments of length $\sigma$. Each segment is labeled by an index $i=1, \ldots, n$ associated with the spherical shell at which it belongs. The pdf associated with all possible paths composed of $n$ segments, being the first segment inside the shell $\mathbf{h}^{\prime}$ and the last one inside the shell $\mathbf{h}$, is defined as

$$
G_{n}\left(\mathbf{h}^{\prime}, \mathbf{h}\right) \equiv \sum^{A} e^{-\sum_{i=1}^{n} U(i)},
$$

in which $\Sigma^{A}$ stands for a sum over all the hypothetical $n$-paths that join the shells $\mathbf{h}^{\prime}$ and $\mathbf{h}$. This function verifies $G_{n}\left(\mathbf{h}^{\prime}, \mathbf{h}\right)=G_{n}\left(\mathbf{h}, \mathbf{h}^{\prime}\right)$. Therefore, the pdf associated with a path of $n+1$ segments may be written as

$$
G_{n+1}\left(\mathbf{h}^{\prime}, \mathbf{h}\right)=\sum^{A}\left(e^{-\Sigma_{i=1}^{n} U(i)} e^{-U(n+1)}\right) .
$$

Assuming $U(n+1)$ to be independent of rest of the segments in the chain and of the starting point of the sequence, the precedent equation reads

$$
G_{n+1}\left(\mathbf{h}^{\prime}, \mathbf{h}\right)=\left(\sum_{\mathbf{h}^{\prime \prime}}{ }^{D} G_{n}\left(\mathbf{h}^{\prime}, \mathbf{h}^{\prime \prime}\right)\right) e^{-U(\mathbf{h})},
$$

where $\Sigma^{D}$ implies a sum over all the $\mathbf{h}^{\prime \prime}$ shells from which we can get into shell $\mathbf{h}$ using a single segment; therefore, shells $\mathbf{h}^{\prime \prime}$ and $\mathbf{h}$ are at a relative distance less or equal to $\sigma$. The above equation stands for the recurrence law needed to calculate the chain pdf once the potential $U(\mathbf{h})$ is given.

We have set the interaction potential $U(i)$ to be proportional to the monomer concentration in shell $i, \phi(i)$,

$$
U(i)=\omega \phi(i),
$$

where $w$ is the excluded volume parameter defined as ${ }^{77}$

$$
\omega=4 \pi \int_{0}^{\infty}\left(1-e^{-u(r)}\right) r^{2} d r .
$$

For the interaction potential $u(r)$ introduced in Eq. (2), we obtain $\omega=4 \pi \sigma^{3} / 3$.

The monomer concentration $\phi(i)$ and the free-end-chain concentration $\epsilon(i)$ are defined as

$$
\begin{aligned}
& \phi(i)=\frac{f}{4 \pi r_{i}^{2} d r} \frac{e^{U(i) \sum_{n=0}^{n=N} \sum_{j=i_{r_{c}}}^{i_{R}} G_{n}\left(i_{r_{c}}, i\right) G_{N-n}(i, j)}}{\sum_{j=i_{r_{c}}}^{i_{R}} G_{N}\left(i_{r_{c}}, j\right)}, \\
& \epsilon(i)=\frac{f}{4 \pi r_{i}^{2} d r} \frac{G_{N}\left(i_{r_{c}}, i\right)}{\sum_{j=i_{r_{c}}}^{i_{i_{N}}} G_{N}\left(i_{r_{c}}, j\right)},
\end{aligned}
$$

where $i_{r_{c}}$ and $i_{R}$ are the shell indices with radius equal to the brush core surface and the cavity wall, respectively. The factor $e^{U(i)}$ in the monomer concentration is introduced to avoid double counting of the interaction term $e^{-U(i)}$ at shell $i$ that comes from splitting the pdf into two terms, one that ends at shell $i$ and the other starting at the same shell.

The change in the free energy when we reduce the cavity size $\Delta \mathcal{F}\left(i_{R}\right)$ is given by

$$
\Delta \mathcal{F}\left(i_{R}\right)=\ln \left(\frac{\Omega\left(i_{R}\right)}{\Omega(\infty)}\right)
$$

being

$$
\Omega(i)=f \sum_{j=i_{r_{c}}}^{i} G_{N}\left(i_{r_{c}}, j\right) .
$$

We have set the reference state as a system with a cavity wall at a distance far enough so that no chain can reach this wall. Therefore, the force to compress the brush, given a cavity size $R$, is

$$
F(R)=-\frac{\partial(\Delta \mathcal{F}(R))}{\partial R} .
$$

Given $R, N$, and $f$, an iterative process is used to obtain the pdf and the density profiles. We have iterated the process until self-consistency is reached. We set the condition for self-consistency such that the sum of the squares of the differences in the density profiles coming from two consecutive iterative steps is less than $10^{-8}$.

The SCF method has the great advantage of being three to four orders of magnitude less expensive in computer time 
than the MC method. In Sec. III we will show that SCF and $\mathrm{MC}$ calculations give similar results for the density profiles $\phi(r)$ and $\epsilon(r)$ in the case of free polymer brushes. However, SCF predictions for the cavity pressure worsen for highly compressed systems.

\section{The Flory theory for polymer solutions}

In the Flory theory the osmotic pressure in a polymer solution of volume $V$ with a degree of polymerization $N$ can be written as

$$
\Pi(R) \propto \frac{-1}{V_{S}}\left(\ln (1-v)+\left(1-\frac{1}{N}\right) v+\chi v^{2}\right),
$$

where $v$ is the volume fraction of solute, $V_{S}=V(1-v)$ is the molar volume of the solvent, and $\chi$ is the Flory parameter. We set $\chi=0$, which is the condition of a dry brush. Under this assumption, the contributions to the free energy come only from the entropy associated with all possible configurations of the system. If we apply this theory to our case, the volume fraction is $v=a N f / V, a=\frac{4}{3} \pi \sigma^{3}$ being the volume of a single monomer. $V$ is the total available cavity volume between the inner wall, represented by the core of the colloidal particle where chains are grafted, and the cavity wall $V(R)=4 \pi / 3\left(R^{3}-r_{c}^{3}\right)$.

At variance with the original Flory treatment, the molar volume of the solvent $V_{S}$ refers to the remaining space in the system once we have subtracted the volume occupied by the monomers; thus, it is no longer a constant value.

The force to compress the cavity will be proportional to the area of the cavity wall and to the change in the osmotic pressure $\Delta \Pi$, thus

$$
F(R) \propto 4 \pi R^{2} \Delta \Pi(R) .
$$

The change in the osmotic pressure is calculated as $\Delta \Pi(R)$ $=\Pi(R)-\Pi^{(\mathrm{nc})}$, where $\Pi(R)$ is given by Eq. $(18)$ and $\Pi^{(\mathrm{nc})}$ is the osmotic pressure for a noncompressed brush. We assume $\Pi^{(\mathrm{nc})}=\Pi\left(R^{*}\right)$, where $R^{*}$ is the radius of the cavity at which $F(R) \rightarrow 0$ for the MC data. The method leaves in Eq. (19) a free constant to be determined. In order to compare the results from Flory theory with MC force profiles we have determined the most suitable value of the constant $(\approx \exp (13.3))$ and this value has been used in all force comparisons.

\section{RESULTS AND DISCUSSION}

We have performed extensive numerical calculations for free and encapsulated spherical polymer brushes and computed the monomer and end-chain density profiles and the compression forces for different values of the number of chains $f$ and the number of monomers per chain $N$. The core radius of the colloidal particle where polymers are grafted is taken to be $r_{c}=5 \sigma$ and is kept constant through all the simulations. The diameter of the monomers is set to $\sigma=1$. We have taken polymer chain lengths in the range of $N=30$ to $N=70$, and we have varied the number of grafted chains from $f=5$ to $f=75$. The range of parameters $(N, f)$ has been chosen in order to obtain chain extents roughly of the same
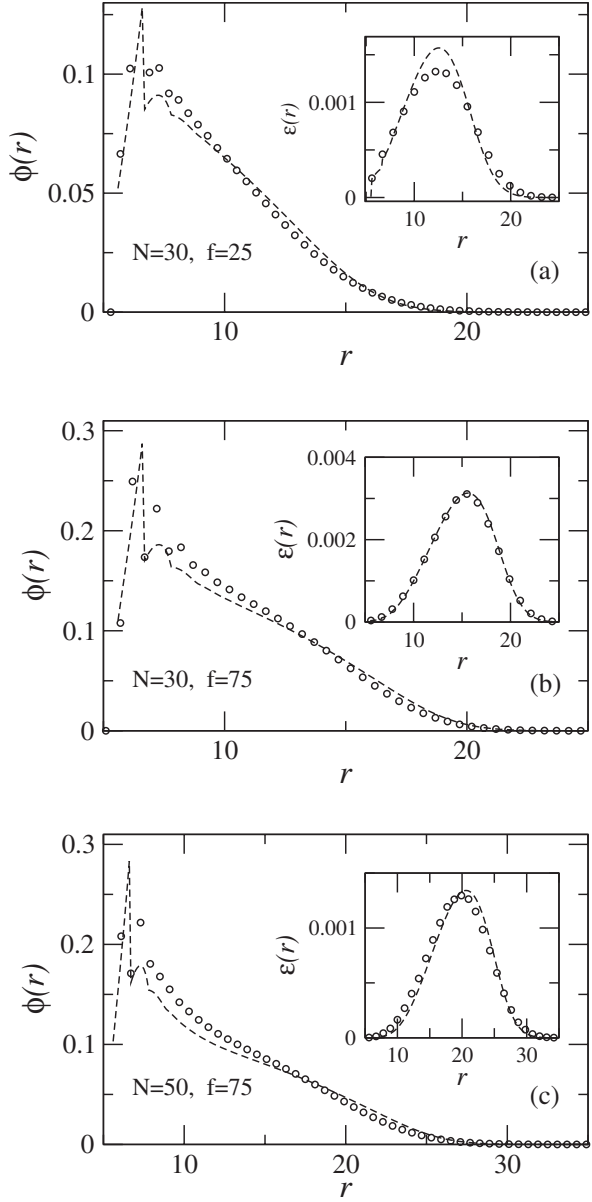

FIG. 2. Comparison between the monomer density profiles $\phi(r)$ for uncompressed spherical brushes obtained with MC $(\mathrm{O})$ and SCF (dashed lines) calculations. Inset: chain-end density profiles. From top to bottom: (a) $N=30, f=25$; (b) $N=30, f=75$; (c) $N=50, f=75$.

order than the diameter of the core where curvature effects are important. For the SCF method we have used a shell thickness $d r=0.1 \sigma$.

\section{A. Density profiles}

We have first studied the monomer and end-chain density profiles $\phi(r)$ and $\epsilon(r)$ for an unconstrained spherical brush. We extend previous MC results ${ }^{32}$ and compare in Figs. 2 and 3 the monomer and end-chain density profiles for unconstrained polymer brushes obtained from our $\mathrm{MC}$ and SCF calculations for different values of $f$ and $N$. The density oscillations observed at small $r$, close to the core of the colloidal particle, are originated due to wall effects of the impenetrable core. In all cases the profiles are roughly similar. In particular, both methods agree very well in the case of the end-chain density profile $\epsilon(r)$ for short chains in a densely packed brush [see inset of Fig. 2(b)]. This is mainly due to the fact that the chains are forced to be mostly fully stretched out and density correlations not present in the SCF formalism are not relevant. On the other hand, the results for the monomer density profiles $\phi(r)$ show systematic differences, although small, between the MC and SCF calculations. Close to the core of the colloidal brush, SCF results display density profiles slightly smaller than those obtained via MC simula- 


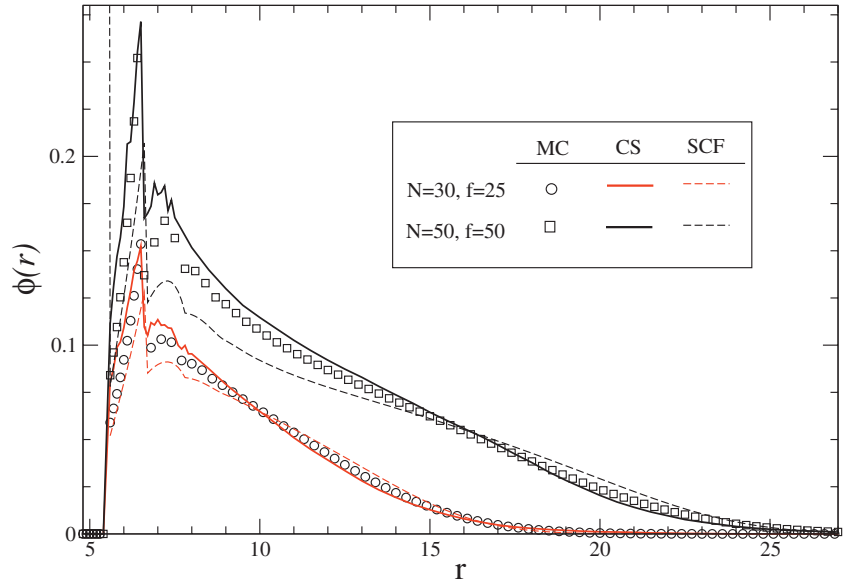

FIG. 3. Comparison between the monomer density profile $\phi(r)$ for free or uncompressed spherical polymer brushes obtained from our MC simulations (symbols) and SCF calculations (dashed lines) for different values of the chain length $N$ and number of grafted chains $f$. The results are compared to the predictions of CS (Ref. 79) (continuous lines).

tions. Also vice versa, in an intermediate region, the SCF method gives densities slightly larger than in the MC simulations. The same systematic behavior was found by Cosgrove et $a l .{ }^{80}$ when comparing MC and SCF density profiles for flat brushes. Cosgrove attributed these differences to the fact that MC simulations account explicitly for the excluded volume effect, whereas SCF accounts only approximately for this effect.

For a free spherical polymer brush Carignano and Szleifer (CS) (Ref. 79) computed the monomer density profile derived from a single-chain mean field theory. A comparison between the CS data and our results is also included in Fig. 3. The better agreement of the CS predictions with our MC simulations, in contrast to the SCF calculations, can be understood in the sense that the CS formalism requires a representative sample of chain configurations as input data to solve the equations that we have generated using our MC method.

\section{B. Cavity pressure and force profiles}

We have measured the force profile exerted by an encapsulated spherical polymer brush onto the external cavity wall through the evaluation of the changes in the free energy due to an infinitesimal change in the radius of the cavity. Within the MC simulations, the force can be calculated by directly measuring the compression probabilities [Eq. (5)]; whereas in the SCF approach, once we have reached self-consistency, we use the pdf in Eqs. (15)-(17).

It is of interest to check to which extent the Flory theory known to be valid for concentrated polymer solutions ${ }^{81}$ without chain-end effects can be applied in such spherically encapsulated polymer systems. That theory has the advantage of being computationally inexpensive. Therefore the predictions arising from Flory theory (see Sec. II C) will be compared to the MC and SCF calculations. The comparison will also serve to check whether the details of the system (spherical geometry and anchored chains) are relevant in the high compression limit.
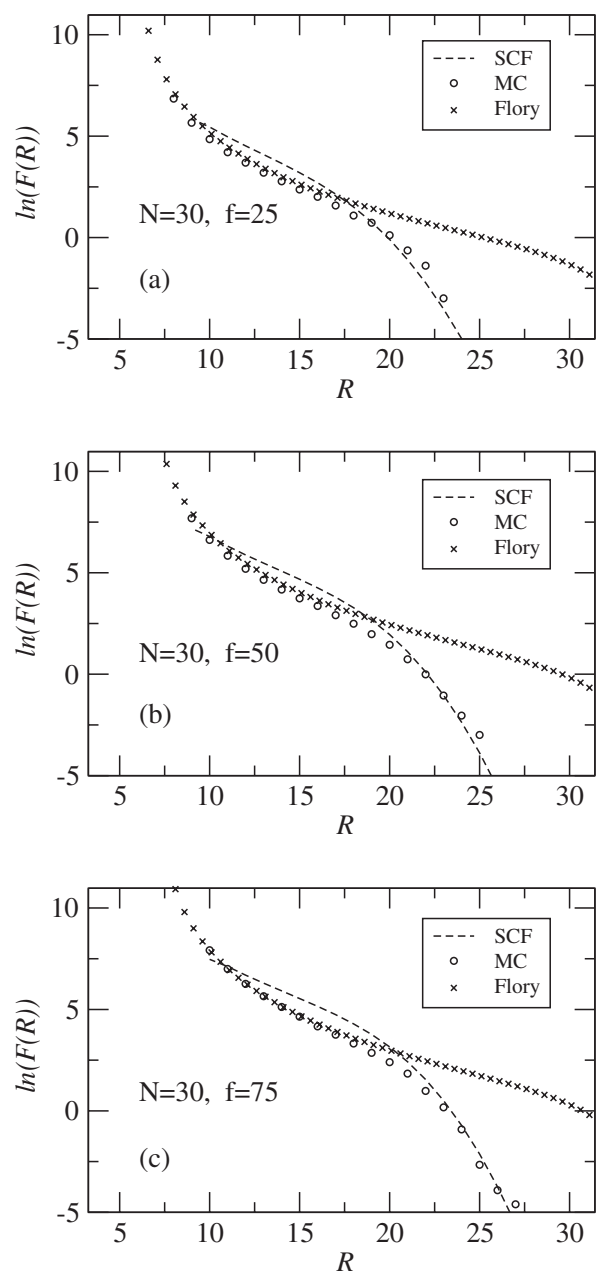

FIG. 4. Semilog plot of the force profile of an encapsulated spherical polymer brush vs the cavity size $R$ for polymer chains of length $N=30$. MC results are represented by filled circles, SCF data by dashed lines, and the predictions coming from the Flory theory by crosses. Different figures stand for different numbers of grafted chains $f$. From top to bottom: (a) $f=25$; (b) $f=50$; (c) $f=75$.

In Figs. 4-6 we present in log-log plots the force profile $F(R)$ versus the cavity size $R$ computed for different values of the polymer chain length $N$ and number of grafted chains $f$. In each figure we include the results coming from the $\mathrm{MC}$ simulations, the predictions of the SCF theory, and results derived from the application of the Flory theory. Figure 4 concentrates on the results derived for short polymer chains $(N=30)$, Fig. 5 for intermediate chain lengths $(N=50)$, and Fig. 6 for long polymers $(N=70)$. In all the cases studied we have used the same fitting constant to adjust the predictions of the Flory theory [see Eq. (19)], and we have taken, as a reference state, a cavity size $R^{*}$ at which $F\left(R^{*}\right) \rightarrow 0$ in the MC simulations.

A direct comparison between SCF and MC force profiles shows a rather good agreement for weakly compressed systems. However, systematic differences are observed for intermediate and high compression values. In the intermediate region we found the SCF forces to be larger than the ones derived from the $\mathrm{MC}$ simulations, whereas for high compressions it is the MC force that becomes larger than the SCF outcome. 

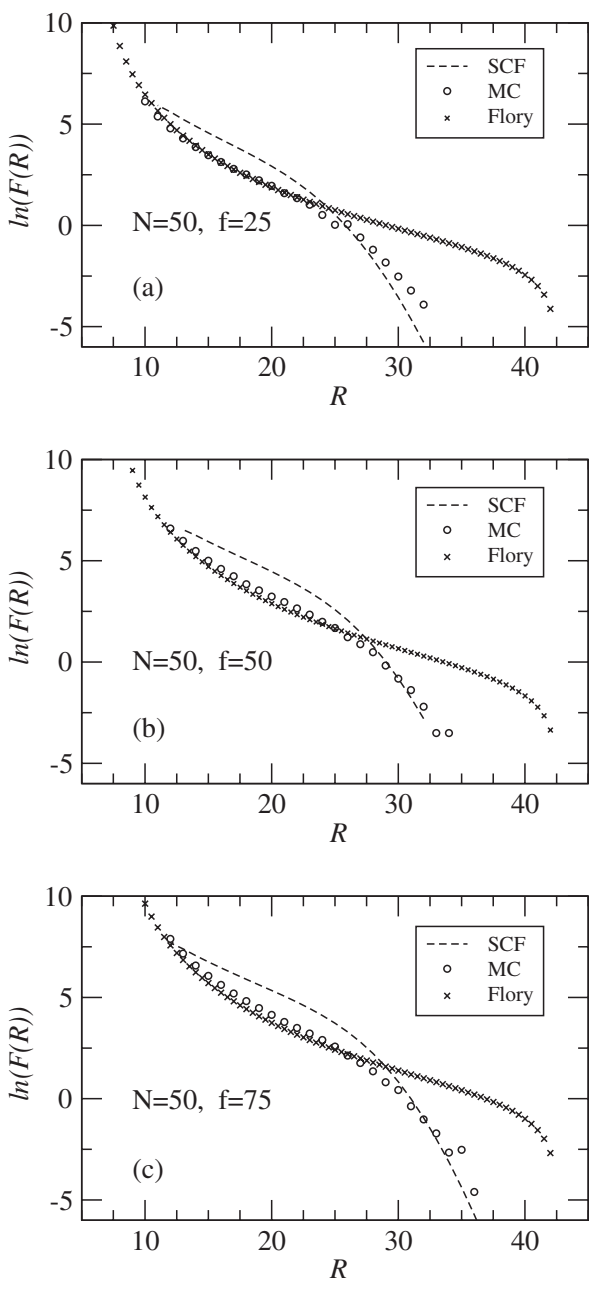

FIG. 5. Same as Fig. 4 for polymer chains of length $N=50$. From top to bottom: (a) $f=25$; (b) $f=50$; (c) $f=75$.

The mismatches observed between the SCF and MC results are due to a twofold effect. For small cavity sizes or highly compressed systems, the assumption in the SCF model of a linear dependence of the mean field potential with the monomer density [see Eq. (11)] breaks down. In fact, under this assumption, the SCF formalism allows a cavity size smaller than the volume occupied by the polymers without requiring an infinite force. Furthermore, for intermediate compression values, the larger forces obtained with the SCF formalism are originated in an overestimation of the monomer interactions. The SCF method does not include the effect of monomer correlations, thus it allows higher average densities in the system than the ones found in the MC simulations. As a consequence, stronger repulsions between the polymers take place and a higher force is required to compress the brush.

The predictions of the Flory theory are found remarkably, despite its simplicity, to be in very good agreement with the results of the MC simulations in the intermediate and high compression regimes. However, the force is overestimated for weakly compressed systems. This result is easily explained since the Flory theory was formerly developed for free polymer chains. As the cavity size grows, the difference between a system of grafted chains and a polymer solution

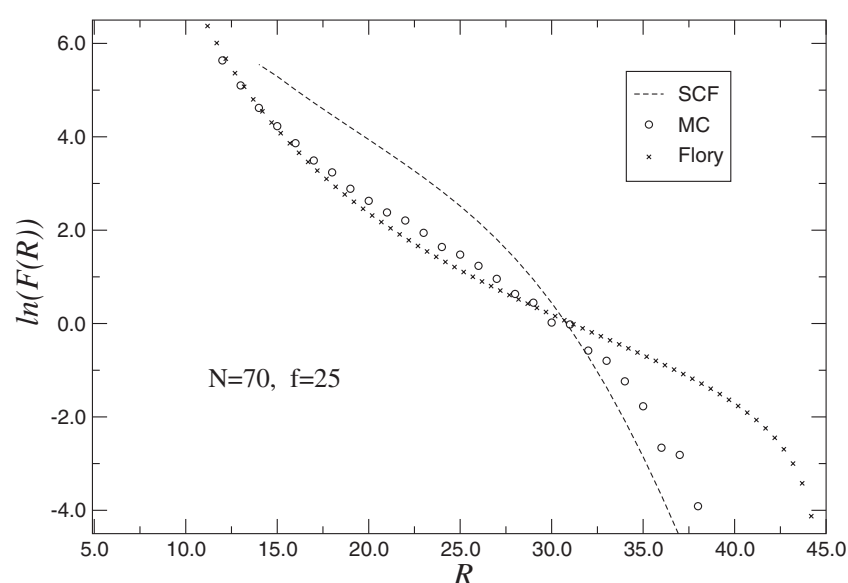

FIG. 6. Same as Fig. 4 for the single case of long polymer brushes with $N=70$ and $f=25$.

becomes evident and, as it is expected, the interaction of the grafted chains with the outer surface is much weaker than the one coming from a polymer solution. It is worth to notice that the MC data can be fitted remarkably well with the Flory theory for intermediate and high compression values, and with the SCF formalism for weakly compressed systems.

We have analyzed the relationship between the monomer volume fraction $v$ and the pressure exerted on the cavity wall $P$ defined as the force to compress the system divided by the area of the cavity. The results for the MC and SCF calculations are shown in log-log plots in Figs. 7 and 8, respectively. In both cases, we find a complex behavior of the monomer volume fraction with the cavity pressure for weakly compressed systems that depends on the different values of the polymer chain length $N$ and number of grafted chains $f$. But, for increasing values of the cavity pressure $v$ and $P$ follow a power law of the form $P \sim v^{\alpha}$ independent of $N$ and $f$. The best fit to the numerical data gives a slope of $\alpha=2.73 \pm 0.04$ for the MC simulations and $\alpha=2.15 \pm 0.05$ for the SCF results. The larger exponent obtained via MC is related to the monomer density correlation effects not ac-

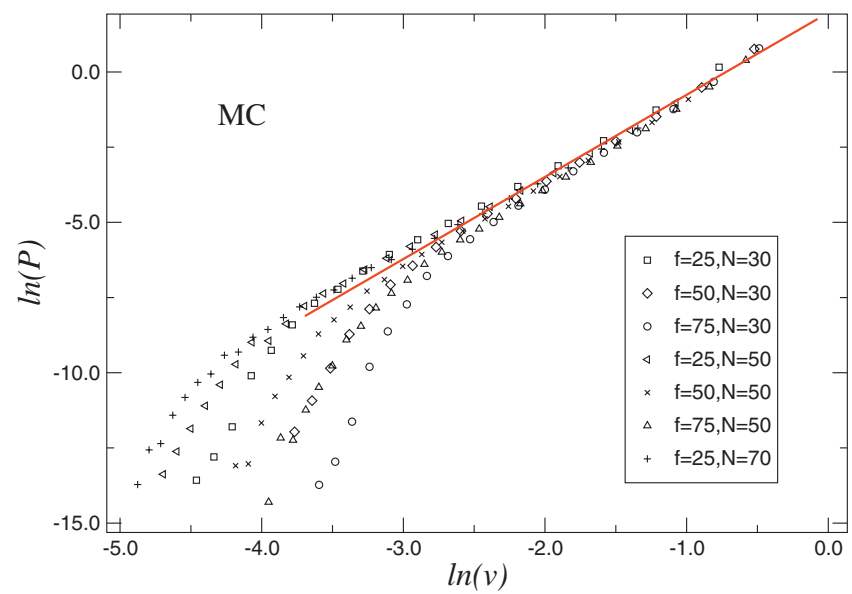

FIG. 7. Log-log plot of the system pressure $P$ vs the monomer volume fraction $v$ obtained from MC simulations for different values of the chain length $N$ and number of grafted chains $f$. A solid line of slope 2.73 is included to guide the eye. 




FIG. 8. Log-log plot of the system pressure vs the monomer volume fraction $v$ derived from the SCF formalism. A solid line of slope 2.15 is included to guide the eye.

counted for in SCF method. One should remark that in both SCF and MC methods, as predicted by polymer theory, at large monomer concentrations all thermodynamic properties reach values that are independent of the degree of polymerization.

It is worth to comment the striking similarity between the exponent obtained via SCF calculations and the des Cloiseaux power law $(\alpha=9 / 4)$ found in semidilute polymer solutions in the limit of very long chains $N \rightarrow \infty .^{78}$ In our systems the chain lengths are quite modest, therefore finite effects are expected to be important. Thus, the coincidence between such exponents is probably fortuitous. Similar accidental coincidences have been observed previously to occur when comparing numerical results and scaling law predictions used out of the expected regime of validity, as for instance in the study of homopolymer adsorption onto a flat wall. $^{82,83}$

Differences between force profiles derived from SCF formalisms and those obtained from other methods that account for chain interdigitation and correlations between the nearest-neighboring bonds are also referenced in several works. For instance, Ruckenstein- $\mathrm{Li}^{84}$ compared the experimental force profile of two interacting crossed cylinders bearing grafted polymer chains, with the numerical data obtained with a generator matrix formalism and from SCF methods. Those authors found the matrix formalism to provide a better agreement with the experimental data than the SCF results. In most cases, the force profiles derived with the matrix formalism and the SCF method are rather similar to the ones we found comparing the MC simulations and the SCF calculation. This reinforces our presumption that interdigitation and monomer correlations are responsible for the observed differences between the MC and SCF data. Additionally, the fact that mean field calculations overestimate the segment-segment interactions in the evaluation of the free energy, as mentioned above, has already been noticed by Lin and Gast. $^{85}$

\section{SUMMARY AND CONCLUDING REMARKS}

In this paper we have studied the behavior of an encapsulated spherical brush inside a spherical cavity. We have measured the monomer density profile and the cavity forces through extensive three-dimensional off-lattice Monte Carlo simulations and using a SCF formalism. In the latter case, we have used directly the pdf recurrence law for the propagator $G_{N}\left(r, r^{\prime}\right)$, avoiding the length scale approximation involved in SCF methods that uses Schrödinger-like equations. Alternatively, we have used a theoretical description based on the Flory theory for polymer solutions to compute the pressure inside the cavity.

A comparison of the predicted forces exerted by the polymer brush onto the cavity surface among the different methods reveals the following. (i) For weakly compressed systems, MC and SCF data show a rather good agreement. However, the force is overestimated in the Flory theory. This difference arises since the Flory theory was developed for free polymer chains and not for polymer brushes; thus, its prediction is not physically relevant when the cavity wall is located at a distance larger than the typical brush extension. (ii) For intermediate and highly compressed systems the MC data agree reasonably well with the results derived from the Flory theory. In the intermediate regime, it is the SCF formalism that overestimates the force. This behavior can be easily explained since the SCF method does not account for monomer correlations, allowing higher monomer densities, and thus higher forces are required to compress the brush. On the other hand, for highly compressed systems, the linear dependence of the mean field potential with the monomer density turns out to be inadequate (the repulsion between monomers allows a volume reduction in the system beyond the own excluded volume of the monomers at a finite energy cost) leading to lower forces than the ones derived from MC simulations.

We have found a power law relationship between the monomer volume fraction and the cavity pressure $P \sim v^{\alpha}$. $\mathrm{SCF}$ data give a slope of $\alpha=2.15$. On the other hand, the MC simulations provide a larger exponent $\alpha=2.73$ that is originated in the monomer correlations not present in the previous models.

The present study of hairy particles trapped inside a confining shell contributes to improve our physical insight about how encapsulated spherical brushes behave. Furthermore it can be of relevance to get a better understanding of particle-filled nanocomposites, the synthesis of hairy particles in mini- and microemulsions, as well as endocytosis processes of drug delivering particles. We expect these results will encourage further theoretical and experimental studies toward the understanding of these confined systems.

\section{ACKNOWLEDGMENTS}

Financial support from MCyT (Spain) and FEDER (EU) (Grant No. FIS2007-60327) is acknowledged. J.J.C. also wants to thank the financial support of MEC (Spain), postdoctoral grant (Grant No. EXP2006-0931). 
${ }^{1}$ S. M. Moghimi, A. C. Hunter, and J. C. Murray, Pharmacol. Rev. 53, 283 (2001).

${ }^{2}$ A. Buxboim, S. S. Daube, and R. Bar-Ziv, Mol. Syst. Biol. 4, 181 (2008).

${ }^{3}$ W. Senaratne, L. Andruzzi, and C. K. Ober, Biomacromolecules 6, 2427 (2005).

${ }^{4}$ M.-C. Daniel and D. Astruc, Chem. Rev. (Washington, D.C.) 104, 293 (2004)

${ }^{5}$ M. Z. Rong, M. Q. Zhang, and W. H. Ruan, Mater. Sci. Technol. 22, 787 (2006).

${ }^{6}$ H. Zou, S. Wu, and J. Shen, Chem. Rev. (Washington, D.C.) 108, 3893 (2008).

${ }^{7}$ D. Li, Q. He, and J. Li, Adv. Colloid Interface Sci. 149, 28 (2009).

${ }^{8}$ I. Luzinov, S. Minko, and V. V. Tsukruk, Soft Matter 4, 714 (2008).

${ }^{9}$ M. Ballauff and Y. Lu, Polymer 48, 1815 (2007).

${ }^{10}$ J. E. Comrie and W. T. S. Huck, Macromol. Rapid Commun. 29, 539 (2008)

${ }^{11}$ P. G. de Gennes, J. Phys. (Paris) 37, 1443 (1976); Macromolecules 13, 1069 (1980); Acad. Sci., Paris, C. R. 300, 839 (1985).

${ }^{12}$ S. Alexander, J. Phys. (Paris) 38, 983 (1977).

${ }^{13}$ A. Chakrabarti and R. Toral, Macromolecules 23, 2016 (1990).

${ }^{14}$ A. Chakrabarti, P. Nelson, and R. Toral, Phys. Rev. A 46, 4930 (1992).

${ }^{15}$ A. Chakrabarti, P. Nelson, and R. Toral, J. Chem. Phys. 100, 748 (1994).

${ }^{16}$ R. Toral, A. Chakrabarti, and R. Dickman, Phys. Rev. E 50, 343 (1994).

${ }^{17}$ J. J. Freire, Adv. Polym. Sci. 143, 35 (1999).

${ }^{18}$ R. R. Netz and D. Andelman, Phys. Rep., Phys. Lett. 380, 95 (2003).

${ }^{19}$ E. P. K. Currie, W. Norde, and M. A. C. Stuart, Adv. Colloid Interface Sci. 100-102, 205 (2003).

${ }^{20}$ A. Naji, C. Seidel, and R. R. Netz, Adv. Polym. Sci. 198, 149 (2006).

${ }^{21}$ Y. Tsujii, K. Ohno, S. Yamamoto, A. Goto, and T. Fukuda, Adv. Polym. Sci. 197, 1 (2006).

${ }^{22}$ R. R. Bhat, M. R. Tomlinson, T. Wu, and J. Genzer, Adv. Polym. Sci. 198, 51 (2006).

${ }^{23}$ R. Descas, J.-U. Sommer, and A. Blumen, Macromol. Theory Simul. 17, 429 (2008).

${ }^{24}$ R. Toomey and M. Tirrell, Annu. Rev. Phys. Chem. 59, 493 (2008).

${ }^{25}$ A. N. Semenov, Sov. Phys. JETP 61, 733 (1985) [Zh. Eksp. Teor. Fiz. 88, $1242(1985)]$

${ }^{26}$ Z. Limpouchova, D. Viduna, and K. Prochazka, Macromolecules 30, 8027 (1997)

${ }^{27}$ K. Jelinek, Z. Limpouchova, and K. Prochazka, Macromol. Theory Simul. 9, 703 (2000)

${ }^{28}$ D. Viduna, Z. Limpouchová, and K. Procházka, J. Chem. Phys. 115, 7309 (2001)

${ }^{29}$ D. I. Dimitrov, A. Milchev, K. Binder, and D. W. Heermann, Macromol. Theory Simul. 15, 573 (2006); D. I. Dimitrov, A. Milchev, and K. Binder, J. Chem. Phys. 125, 034905 (2006).

${ }^{30}$ M. Kruk, B. Dufour, E. B. Celer, T. Kowalewski, M. Jaroniec, and K. Matyjaszewski, Macromolecules 41, 8584 (2008).

${ }^{31}$ C. Pastorino, K. Binder, and M. Muller, Macromolecules 42, 401 (2009).

${ }^{32}$ R. Toral and A. Chakrabarti, Phys. Rev. E 47, 4240 (1993).

${ }^{33}$ E. B. Zhulina, T. M. Birshtein, and O. V. Borisov, Eur. Phys. J. E 20, 243 (2006).

${ }^{34}$ S. S. Sheiko, B. S. Sumerlin, and K. Matyjaszewski, Prog. Polym. Sci. 33, 759 (2008).

${ }^{35}$ J. U. Kim and M. W. Matsen, Macromolecules 42, 3430 (2009).

${ }^{36}$ U. Wattendorf and H. P. Merkle, J. Pharm. Sci. 97, 4655 (2008).

${ }^{37}$ D. H. Napper, Polymeric Stabilization of Colloidal Dispersion (Academic, New York, 1983)

${ }^{38}$ W. B. Russel, D. A. Saville, and W. R. Schowalter, Colloidal Dispersions (Cambridge University Press, Cambridge, England, 1989).

${ }^{39}$ S. Phan, W. B. Russel, Z. Cheng, J. Zhu, P. M. Chaikin, J. H. Dunsmuir, and R. H. Ottewill, Phys. Rev. E 54, 6633 (1996).

${ }^{40}$ J. A. Opsteen, R. P. Brinkhuis, R. L. M. Teeuwen, D. W. P. M. Löwik, and J. C. M. van Hest, Chem. Commun. (Cambridge) 30, 3136 (2007).

${ }^{41}$ M. Ballauff, Prog. Polym. Sci. 32, 1135 (2007).

${ }^{42}$ Y. Mei, M. Hoffmann, M. Ballauff, and A. Jusufi, Phys. Rev. E 77, 031805 (2008).
${ }^{43}$ N. P. Shusharina and M. Rubinstein, Macromolecules 41, 203 (2008).

${ }^{44}$ J. K. Wolterink, F. A. M. Leermakers, G. J. Fleer, L. K. Koopal, E. B. Zhulina, and O. V. Borisov, Macromolecules 32, 2365 (1999).

${ }^{45}$ N. Dan and M. Tirell, Macromolecules 25, 2890 (1992).

${ }^{46}$ R. Israels, F. A. M. Leermakers, and G. J. Fleer, Macromolecules 27, 3087 (1994).

${ }^{47}$ K. Jelinek, F. Uhlik, Z. Limpouchova, and K. Procházka, J. Phys. Chem. B 107, 8241 (2003).

${ }^{48}$ J.-R. Roan, Int. J. Mod. Phys. B 18, 2469 (2004).

${ }^{49}$ F. A. M. Leermakers, E. B. Zhulina, J. van Male, A. A. Mercurieva, G. J. Fleer, and T. M. Birshtein, Langmuir 17, 4459 (2001).

${ }^{50}$ K. Jelínek, Z. Limpouchová, F. Uhlík, and K. Procházka, Macromolecules 40, 7656 (2007)

${ }^{51}$ F. Uhlík, K. Jelínek, Z. Limpouchová, and K. Procházka, Macromolecules 41, 3711 (2008).

${ }^{52}$ M. H. Nommensen, G. Duits, D. van den Ende, and J. Mellema, Phys. Rev. E 59, 3147 (1999).

${ }^{53}$ G. S. Grest and M. Murat, Macromolecules 26, 3108 (1993).

${ }^{54}$ F. Uhlík, Z. Limpouchová, K. Jelínek, and K. Procházka, J. Chem. Phys. 118, 11258 (2003); J. Chem. Phys. 121, 2367 (2004).

${ }^{55}$ A. S. Almusallam and D. S. Sholl, Nanotechnology 16, S409 (2005).

${ }^{56}$ C. N. Likos, R. Blaak, and A. Wynveen, J. Phys.: Condens. Matter 20, 494221 (2008).

${ }^{57}$ R. Ni, D. P. Cao, W. C. Wang, and A. Jusufi, Macromolecules 41, 5477 (2008).

${ }^{58}$ S. Huissmann, R. Blaak, and C. N. Likos, Macromolecules 42, 2806 (2009).

${ }^{59}$ M. Daoud and J. P. Cotton, J. Phys. (Paris) 43, 531 (1982).

${ }^{60}$ T. A. Witten and P. A. Pincus, Macromolecules 19, 2509 (1986).

${ }^{61}$ C. M. Wijmans, F. A. M. Leermakers, and G. J. Fleer, Langmuir 10, 4514 (1994).

${ }^{62}$ I. Borukhov and L. Leibler, Phys. Rev. E 62, R41 (2000).

${ }^{63}$ J. J. Cerdà, T. Sintes, and R. Toral, Macromolecules 36, 1407 (2003).

${ }^{64}$ A. Striolo, Phys. Rev. E 74, 041401 (2006).

${ }^{65}$ C. N. Likos, Soft Matter 2, 478 (2006).

${ }^{66}$ C. Mayer and C. N. Likos, Macromolecules 40, 1196 (2007).

${ }^{67}$ A. Striolo, Nanotechnology 19, 445606 (2008).

${ }^{68}$ P. Voudouris, J. Choi, H. Dong, M. R. Bockstaller, K. Matyjaszewski, and G. Fytas, Macromolecules 42, 2721 (2009).

${ }^{69}$ P. Romiszowski and A. Sikorski, J. Mol. Model. 15, 681 (2009) and references therein.

${ }^{70}$ K. Procházka, T. J. Martin, S. E. Webber, and P. Munk, Macromolecules 29, 6526 (1996).

${ }^{71}$ L. Bombalski, K. Min, H. Dong, C. Tang, and K. Matyjaszewski, Macromolecules 40, 7429 (2007).

${ }^{72}$ K. Min, H. Gao, J. A. Yoon, W. Wu, T. Kowalewski, and K. Matyjaszewski, Macromolecules 42, 1597 (2009).

${ }^{73}$ K. Landfester, Annu. Rev. Mater. Res. 36, 231 (2006).

${ }^{74}$ A. Baungärtner, in Applications of the Monte Carlo Method in Statistical Physics, Topics in Current Physics Vol. 36, 2nd ed., edited by K. Binder (Springer-Verlag, Berlin, 1987).

${ }^{75}$ M. Allen and D. Tildesley, Computer Simulation of Liquids (Clarendon, Oxford, 1987)

${ }^{76}$ R. Dickman, J. Chem. Phys. 87, 2246 (1987).

${ }^{77}$ S. F. Edwards, Proc. Phys. Soc. London 85, 613 (1965).

${ }^{78}$ P. G. de Gennes, Scaling Concepts in Polymer Physics (Cornell University, Ithaca, 1979).

${ }^{79}$ M. A. Carignano and I. Szleifer, J. Chem. Phys. 102, 8662 (1995).

${ }^{80}$ T. Cosgrove, T. Heath, B. van Lent, F. A. M. Leermakers, and J. M. H. M. Scheutjens, Macromolecules 20, 1692 (1987).

${ }^{81}$ P. J. Flory, Principles of Polymer Chemistry (Cornell University Press, Ithaca, 1953).

${ }^{82}$ J. de Joannis, C.-W. Park, J. Thomatos, and I. A. Bitsanis, Langmuir 17, 69 (2001).

${ }^{83}$ J. B. Avalos, A. D. Mackie, and S. Díez-Orrite, Macromolecules 37, 1143 (2004)

${ }^{84}$ E. Ruckenstein and B. Li, J. Chem. Phys. 107, 932 (1997).

${ }^{85}$ E. K. Lin and A. P. Gast, Macromolecules 29, 390 (1996). 